Lu Shipan, Library, Teachers College, Jimei University, Xiamen 361021, China

\title{
NOTES ON THE APPROXIMATELY CONTINUOUS HENSTOCK INTEGRAL
}

\begin{abstract}
There are two different definitions about the approximately continuous Henstock integral or $A H$ integral, for short. In this paper we prove that they are equivalent, and that the $A H$ integral is included in the $A D$ integral defined by Kubota in [4]. Also we give a solution for the problem put forward by Gordon in [3] (p. 255).
\end{abstract}

\section{The equivalence of the two definitions about the $A H$ integral}

Gordon defines the $A H$ integral in [3] as follows.

Definition 1.1 A collection $\Delta$ of tagged intervals in $[a, b]$ is an approximate full cover of $E \subset[a, b]$ if for each $x \in E$ there exists a measurable set $S_{x} \subset[a, b]$ such that $x \in S_{x}^{d}$ and $([c, d], x) \in \Delta$ if and only if $c, d \in S_{x}$, where $S_{x}^{d}$ represents the set of all $y \in S_{x}$ such that $y$ is a point of density of $S_{x}$.

Definition 1.2 A function $f:[a, b] \mapsto \mathbb{R}$ is ap-Henstock integrable on $[a, b]$ if there exists a real number $A$ with the following property: for each $\epsilon>0$ there exists an approximate full cover $\Delta$ of $[a, b]$ such that $|f(\mathcal{P})-A|<\epsilon$ whenever $\mathcal{P}$ is a tagged partition of $[a, b]$ that is a subset of $\Delta$, where $f(\mathcal{P})=$ $(\mathcal{P}) \sum f(x) \mu(I)$ whenever $\mathcal{P}=\{(I, x)\}$.

Gordon gives another definition about the $A H$ integral in [2], which is called $S$-Henstock integral.

\footnotetext{
Key Words: $A H$ integral, $A D$ integral

Mathematical Reviews subject classification: 26A39

Received by the editors November 5,1995

* This work was done while the author was a visiting scholar at the Mathematics Institute of Yunnan University. I would like to thank the referee for the helpful advice.
} 
Definition 1.3 The collection of measurable sets $\left\{S_{x}: x \in E\right.$ and $x \in S_{x} \cap$ $\left.S_{x}^{d}\right\}$ is called an approximate distribution on $E$. Let $\delta$ be a positive function. A partial tagged partition $\mathcal{P}=\{(I, x)\}$ is called a $S_{\delta}$ partition on $E$ if $x \in E$, $x \in I \subset(x-\delta(x), x+\delta(x))$ and the two extreme points of $I$ belong to $S_{x}$.

Definition 1.4 A function $f:[a, b] \mapsto \mathbb{R}$ is ap-Henstock integrable on $[a, b]$ if there exists a real number $A$ and an approximate distribution $\left\{S_{x}\right\}$, such that for every $\epsilon>0$ there exists $\delta(x)>0$ such that $|f(\mathcal{P})-A|<\epsilon$ whenever $\mathcal{P}$ is $a S_{\delta}$ partition on $[a, b], \mathcal{P}=\{(I, x)\}$.

The difference between Definition 1.2 and Definition 1.4 is that $\left\{S_{x}\right\}$ of the latter is independent of $\epsilon$, whose property is much stronger. In order to show the equivalence between them, it is sufficient to show the following proposition.

Proposition 1.1 Under the assumption of Definition 1.2, we can construct an approximate distribution $\left\{S_{x}\right\}$ which satisfies Definition 1.4.

Proof. Let $\epsilon=2^{-n}$. Then there exists a corresponding approximate full cover $\Delta_{n}$. Therefore, for each $n$, there exists an approximate distribution $\left\{S_{x, n}\right\}$ which corresponds to $\Delta_{n}$. Assume that $\Delta_{n+1} \subset \Delta_{n}$, i.e., $S_{x, n+1} \subset S_{x, n}$, $n=1,2, \ldots$.

Since $x$ is a point of density of $S_{x, n}$, there exists $h_{x, n}>0$ such that $\mu\left(S_{x, n} \cap(x-h, x+h)\right) \geq 2 h\left(1-2^{-n}\right)$, whenever $0<h<h_{x, n}$. We can assume that $h_{x, n}$ is monotone decreasing and tends to zero. Let

$$
S_{x}=\{x\} \cup\left(\cup_{n=1}^{\infty}\left(\left(\left(x-h_{x, n}, x+h_{x, n}\right) \backslash\left(x-h_{x, n+1}, x+h_{x, n+1}\right)\right) \cap S_{x, n}\right)\right) .
$$

Then we can show that $x \in S_{x} \cap S_{x}^{d}$. In fact, for any $h>0$, there is a positive integer $i$ such that $h_{i+1}<h \leq h_{i}$. Then

$$
\begin{gathered}
\mu\left(S_{x} \cap(x-h, x+h)\right)=\mu\left(\left((x-h, x+h) \backslash\left(x-h_{x, i+1}, x+h_{x, i+1}\right)\right) \cap S_{x, i}\right) \\
\cup\left(\cup_{n=i+1}^{\infty}\left(\left(\left(x-h_{x, n}, x+h_{x, n}\right) \backslash\left(x-h_{x, n+1}, x+h_{x, n+1}\right)\right) \cap S_{x, n}\right)\right) \\
\geq\left(2 h\left(1-2^{-i}\right)-2 h_{i+1}\right)+\sum_{n=i+1}^{\infty}\left(2 h_{n}\left(1-2^{-n}\right)-2 h_{n+1}\right) \geq \\
2 h\left(1-2^{-i}\right)-\sum_{n=i+1}^{\infty} 2 h_{n} \cdot 2^{-n} \geq 2 h\left(1-2^{-i}\right)-2 h \cdot 2^{-i}=2 h\left(1-2^{-i+1}\right)
\end{gathered}
$$

which implies that

$$
\lim _{h \rightarrow 0} \frac{\mu\left(S_{x} \cap(x-h, x+h)\right)}{2 h}=1
$$


that is $x \in S_{x}^{d}$. Since $x \in S_{x}$ is obviously satisfied, so $x \in S_{x} \cap S_{x}^{d}$.

Let $0<\epsilon<1 / 2$ be given and let $\epsilon_{n} \leq \epsilon<\epsilon_{n-1}$. We can choose $\delta_{(x)}=$ $h_{x, n}$. Then $S_{x} \cap\left(x-h_{x, n}, x+h_{x, n}\right) \subset S_{x, n}$. Therefore, for each $S_{\delta}$ partition $\mathcal{P}=\{(I, x)\}$, we have $|f(\mathcal{P})-A|<\epsilon_{n} \leq \epsilon$.

In the same fashion, we can convert the definition of $A C G_{\Delta}$ defined by Gordon ([3], p. 252) into the following one.

Definition 1.5 Let $F:[a, b] \mapsto \mathbb{R}$ be measurable and let $E \subset[a, b]$ be measurable. The function $F$ is $A C_{\Delta}$ on $E$ if there exists an approximate distribution $\left\{S_{x}\right\}$ on $E$ such that for each $\epsilon>0$ there exist a positive number $\eta$ and a positive function $\delta$ such that $|F(\mathcal{P})|<\epsilon$ for each $S_{\delta}$ partial partition $\mathcal{P}$ of $E$ that satisfies $\mu(\mathcal{P})<\eta$. The function $F$ is $A C G_{\Delta}$ on $E$ if $E$ can be expressed as a countable union of measurable sets on each of which the function $F$ is $A C_{\Delta}$.

$2 A H \subset A D$

Definition 2.1 (Kubota) ([4]). A function $f:[a, b] \mapsto \mathbb{R}$ is AD-integrable on $[a, b]$ if there exists a function $F$ such that

(1) $F$ is approximately continuous on $[a, b]$,

(2) $F$ is $[A C G]$ on $[a, b]$ (i.e., $[a, b]$ can be expressed as a countable union of closed sets, on each of which $F$ is $A C$ ),

(3) $F_{a p}^{\prime}(x)=f(x)$ a.e. on $[a, b]$.

Now we will prove that the $A H$ integral is included in $A D$.

Theorem 2.1 $A H \subset A D$.

Proof. We only need to prove that $F$, the primitive function of $f$ is $[A C G]$. Since $F$ is $A C G_{\Delta}$ on $[a, b]$, there exist $E_{i}$ such that $[a, b]=\cup_{i} E_{i}$ and $F$ is $A C_{\Delta}$ on each $E_{i}$. Therefore there exists an approximate distribution $\left\{S_{x}: x \in E_{i}\right\}$ on $E_{i}$. Let $\epsilon=1$ be given. Then there exist $\eta>0$ and $\delta^{*}(x)>0$ such that for every $S_{\delta^{*}}$ partial partition $\mathcal{P}=\left\{(I, x): x \in E_{i}\right\}$ and $\mu(\mathcal{P})<\eta$, we have $|F|(\mathcal{P})<1$.

Since $x \in S_{x}^{d}$, there exists $\delta(x)>0\left(\delta(x) \leq \delta^{*}(x)\right)$ on $E_{i}$, such that $\mu\left(S_{x} \cap\right.$ $J)>(1 / 2) \mu(J)$ whenever the interval $J$ satisfies $x \in J \subset(x-\delta(x), x+\delta(x))$. Let $E_{i j}=\left\{x \in E_{i}: \delta(x)>1 / j\right\}$, where $j>1 / \eta$. Then $E_{i}=\cup_{j} E_{i j}$. Let $E_{i j k}=E_{i j} \cap[(k-1) / j, k / j]$. Then $E_{i j}=\cup_{k=-\infty}^{+\infty} E_{i j k}$. For convenience sake, we denote $E_{i j k}$ by $E$. 
It is known ([3], p. 381) that $F$ is $V B$ on $E$. Next we will prove that $F_{/ \bar{E}}$ is continuous. Suppose that $F_{\bar{E}}$ is not continuous at some point $c \in \bar{E}$. Then there exists a strictly monotone sequence $\left\{c_{n}\right\}$ in $E$ such that $\lim _{n \rightarrow \infty} c_{n}=c$ and $\lim _{n \rightarrow \infty} F\left(c_{n}\right)$ exists (perhaps infinite) but does not equal $F(c)$. We will consider the case in which $\left\{c_{n}\right\}$ is an increasing sequence (the other case is similar) and $\lim _{n \rightarrow \infty} F\left(c_{n}\right)=A \neq F(c)$. Set $B=|A-F(c)|$. Then there exists a positive integer $N$ such that $\left|F\left(c_{n}\right)-F(c)\right|>B / 2$, for all $n>N$. Since $F$ is approximately continuous at $c$, there exist $S_{c}$ of density 1 at $c$ and $\delta>0\left(\delta<1 / j, \delta<c-c_{N}\right)$ such that

$$
|F(x)-F(c)|<\frac{B}{4}, \text { for } x \in S_{c} \cap(c-\delta, c)
$$

and

$$
\frac{\mu\left(S_{c} \cap(c-h, c)\right)}{h}>\frac{1}{2}, \text { whenever } 0<h<\delta .
$$

We can assume that $\left\{c_{n}\right\} \subset(c-\delta, c)$. Select a subsequence of $\left\{c_{n}\right\}$ (again denoted by $\left.\left\{c_{n}\right\}\right)$ and a sequence $\left\{x_{n}\right\}$ such that $c_{1}<x_{1}<c_{2}<x_{2}<\cdots<$ $c_{n}<x_{n}<\cdots$, and $x_{n} \in S_{c_{n}} \cap S_{c} \cap\left(c_{n}, c\right)$. This is possible because the following inequalities hold

$$
\frac{\mu\left(S_{c_{n}} \cap\left(c_{n}, c\right)\right)}{c-c_{n}}>\frac{1}{2} \text { and } \frac{\mu\left(S_{c} \cap\left(c_{n}, c\right)\right)}{c-c_{n}}>\frac{1}{2} .
$$

For each $n$, we have $\left|F\left(x_{n}\right)-F(c)\right|<B / 4$. Thus

$$
\left|F\left(c_{n}\right)-F\left(x_{n}\right)\right| \geq\left|F\left(c_{n}\right)-F(c)\right|-\left|F(c)-F\left(x_{n}\right)\right| \geq \frac{B}{2}-\frac{B}{4}=\frac{B}{4} .
$$

Therefore, it follows that $\sum_{n=1}^{\infty}\left|F\left(c_{n}\right)-F\left(x_{n}\right)\right| \geq \sum_{n=1}^{\infty} B / 4=+\infty$, which contradicts $\sum\left|F\left(c_{n}\right)-F\left(x_{n}\right)\right| \leq 1$. Thus, $F_{/ E}$ is continuous.

Hence $F$ is $V B$ on $\bar{E}$ ([5], p. 221). Since $F$ satisfies Lusin's condition $(N)$ ([3], p. 380 or [1]), $F$ is $A C$ on $\bar{E}$ ([5], p. 227). From $[a, b]=\cup_{i j k} \bar{E}$ it follows that $F \in[A C G]$. This completes the proof.

Remark 2.1 Kubota affirms that AP (Burkill's approximately continuous Perron integral) is included in $A D$. Though his proof had some errors, his conclusion is correct, because $A P_{o} \subset A P=A H \subset A D$.

Remark 2.2 After citing the example "there exists a function $F$, which is $A C G$, but not $A C G_{\Delta}$ " given by Tolstoff, Gordon points out that "although this is the case, we still can not prove that $F^{\prime}$ is not ap-Henstock integrable". 
Now, we can answer the question explicitly. Because both the Khintchine integral and the $A H$ integral are contained in the $A D$ integral, and it is easy to show that the value of the AD integral is unique (see [4]). Therefore, from this example, we already know that $F^{\prime}$ can not be $A H$ integrable. If $F^{\prime}$ is $A H$ integrable then its primitive function is $F$. But this is not the case.

\section{References}

[1] S. Fu, S-Henstock integration and the approximately strong Lusin condition, Real Anal. Exchange 19 (1993-1994), 312-316.

[2] R. Gordon, The inversion of approximate and dyadic derivatives using an extension of the Henstock integral, Real Anal. Exchange 16 (1990-1991), $154-168$.

[3] R. Gordon, The integrals of Lebesgue, Denjoy, Perron and Henstock, AMS (1994).

[4] Y. Kubota, An integral of Denjoy type, Proc. Japan Acad 40 (1964), 713717.

[5] S. Saks, Theory of the integral, 2nd. rev. ed., vol. PWN, Monografie Matematyczne, Warsaw, 1937. 